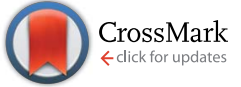

Cite this: Chem. Sci., 2017, 8, 953

Received 24th August 2016

Accepted 24th September 2016

DOI: $10.1039 / c 6 s c 03793 c$

www.rsc.org/chemicalscience

\section{Pyrimidine-based twisted donor-acceptor delayed fluorescence molecules: a new universal platform for highly efficient blue electroluminescence $\uparrow$}

\author{
In Seob Park, ${ }^{\text {ab }}$ Hideaki Komiyama ${ }^{a}$ and Takuma Yasuda*ab
}

Deep-blue emitters that can harvest both singlet and triplet excited states to give high electron-to-photon conversion efficiencies are highly desired for applications in full-color displays and white lighting devices based on organic light-emitting diodes (OLEDs). Thermally activated delayed fluorescence (TADF) molecules based on highly twisted donor-acceptor (D-A) configurations are promising emitting dopants for the construction of efficient deep-blue OLEDs. In this study, a simple and versatile D-A system combining acridan-based donors and pyrimidine-based acceptors has been developed as a new platform for high-efficiency deep-blue TADF emitters. The designed pre-twisted acridan-pyrimidine D-A molecules exhibit small singlet-triplet energy splitting and high photoluminescence quantum yields, functioning as efficient deep-blue TADF emitters. The OLEDs utilizing these TADF emitters display bright blue electroluminescence with external quantum efficiencies of up to $20.4 \%$, maximum current efficiencies of $41.7 \mathrm{~cd} \mathrm{~A}^{-1}$, maximum power efficiencies of $37.2 \mathrm{~lm} \mathrm{~W}^{-1}$, and color coordinates of $(0.16$, 0.23). The design strategy featuring such acridan-pyrimidine D-A motifs can offer great prospects for further developing high-performance deep-blue TADF emitters and TADF-OLEDs.

\section{Introduction}

Organic light-emitting diodes (OLEDs) have made great progress towards applications in next-generation flat-panel displays and solid-state lighting over the past three decades since the pioneering work of Tang and VanSlyke in $1987 .^{1}$ To produce fullcolor displays and white lighting devices based on OLED technologies, the three primary RGB (red, green, and blue) colors are indispensable. Up to date, red and green phosphorescent emitters based on organometallic iridium or platinum complexes primarily match the requirements of application in terms of efficiency, stability, and color purity. ${ }^{2-5}$ However, the overall device performance of blue (especially deep-blue) OLEDs, based on phosphorescent emitters ${ }^{6}$ or conventional fluorescent emitters, ${ }^{7}$ still lags behind its red and green counterparts. Hence, further improvement in the electroluminescence (EL) efficiency, operational stability, and color index is required. Driven by such technological demands, it is vital to develop highly efficient deep-blue emitters with Commission Internationale de

${ }^{a}$ INAMORI Frontier Research Center (IFRC), Kyushu University, 744 Motooka, Nishi-ku, Fukuoka 819-0395, Japan.E-mail: yasuda@ifrc.kyushu-u.ac.jp

${ }^{b}$ Department of Applied Chemistry, Graduate School of Engineering, Kyushu University, 744 Motooka, Nishi-ku, Fukuoka 819-0395, Japan

$\dagger$ Electronic supplementary information (ESI) available: General methods, synthesis and characterization data for intermediates, additional computational and photophysical data, TGA data, and OLED device characteristics. CCDC 1500786. For ESI and crystallographic data in CIF or other electronic format see DOI: $10.1039 / \mathrm{c} 6 \mathrm{sc} 03793 \mathrm{c}$
l'Éclairage chromaticity coordinate $\left(\mathrm{CIE}_{x, y}\right)$ values below 0.15 , matching closely with the National Television System Committee (NTSC) standard pure blue coordinates of $(0.14,0.08)$.

Over the last few years, OLEDs utilizing thermally activated delayed fluorescence (TADF) emitters, which can harvest both triplet $\left(\mathrm{T}_{1}\right)$ and singlet $\left(\mathrm{S}_{1}\right)$ excitons for EL via efficient reverse intersystem crossing (RISC), have shown conspicuous improvement in device efficiencies, achieving internal EL quantum efficiencies $\left(\eta_{\text {int }}\right)$ of nearly $100 \% .^{8-14}$ Replacing phosphorescent organometallic emitters with efficient metal-free pure-organic TADF emitters offers the possibility to not only reduce the cost of materials by eliminating the need for expensive precious metals but also to solve the stability issue of the existing blue phosphorescent materials and their devices. In general, TADF emitters are composed of electron donor (D) and acceptor (A) moieties, which give rise to a small spatial overlap between the highest occupied molecular orbital (HOMO) and the lowest unoccupied molecular orbital (LUMO) in order to minimize the singlet-triplet energy splitting $\left(\Delta E_{\mathrm{ST}}\right)$ and thereby accelerate the RISC process from its non-radiative $T_{1}$ to radiative $S_{1}$ states. Based on this design principle, various $\mathrm{D}-\mathrm{A}$ and D-A-D structured blue/sky-blue TADF emitters containing triazine, ${ }^{9,15-17}$ benzosulfone, ${ }^{\mathbf{1 0 , 1 8 - 2 2}}$ phenone, ${ }^{\mathbf{2 3 - 2 6}}$ benzonitrile, ${ }^{8,27-32}$ or phenylborane ${ }^{33-37}$ as the A moiety have recently been synthesized and applied in TADF-OLEDs. However, highperformance blue TADF emitters are still very rare and only a few of them can achieve both a high external EL quantum efficiency $\left(\eta_{\text {ext }}\right)$ exceeding $20 \%$ and a suitable color purity with 
a $\mathrm{CIE}_{y}$ value below $0.25 .^{10,16,21,22,29,33,34,38}$ Hence, it remains quite challenging to search for an appropriate combination of $\mathrm{D}$ and A moieties to simultaneously attain both excellent EL efficiency and high color purity for deep-blue TADF materials.

Herein, we report a new family of highly efficient deep-blue TADF emitters based on a simple pre-twisted D-A architecture (Fig. 1) in which a pyrimidine-based acceptor moiety is connected with a spiroacridan/acridan-based donor moiety through a phenylene $\pi$-spacer. Owing to the large steric repulsion between the hydrogen atoms of the acridan unit and the adjacent phenylene spacer, this D-A system offers nearly orthogonal conformations in the ground $\left(\mathrm{S}_{0}\right)$ and $\mathrm{S}_{1}$ states, leading to an effective spatial separation of the HOMO and LUMO and a reduction in $\Delta E_{\mathrm{ST}}$. Hence it enables efficient upconversion from the $T_{1}$ to the $S_{1}$ state. We envisage that the pyrimidine unit can serve as a universal building block for deepblue TADF materials as it possesses a weaker electron-accepting nature than the widely used triazine unit and thus increases the bandgap energy $\left(E_{\mathrm{g}}\right)$ and $\mathrm{S}_{1}$ and $\mathrm{T}_{1}$ energy levels of the resulting D-A molecules. Moreover, the pyrimidine unit can be substituted with a variety of functional groups and fine-tuning of the photophysical and electronic properties can be achieved with simple chemical modifications.

\section{Results and discussion}

\section{Molecular design and synthesis}

As shown in Fig. 2, we designed a new series of D-A molecules, 1-5 consisting of 2,4,6-triphenylpyrimidine (PPM) or 2-phenylpyrimidine (PM) as an acceptor and spiro[2,7-dimethylacridan9,9'-fluorene] (MFAc), spiro[2,7-dimethylacridan-9,9'-xanthene] (MXAc), or 9,9-dimethylacridan (Ac) as a donor. The selection of the PPM and PM units which have relatively weak electronwithdrawing characteristics and intrinsic high $\mathrm{T}_{1}$ energies is key to producing wide-bandgap deep-blue TADF materials. Our design strategy is justified by time-dependent density functional theory (TD-DFT) calculations, which provide insights into the geometrical and electronic properties of 1-5 at the molecular level. As can be seen from Fig. 2, all of these molecules adopt highly twisted D-A conformations in their optimized geometries, with dihedral angles between the acridan unit and the adjacent phenylene ring $\left(\theta_{1}\right)$ of $87-90^{\circ}$ owing to the steric repulsion arising from their peri-hydrogen atoms. Meanwhile, the dihedral angles between the pyrimidine ring and the central

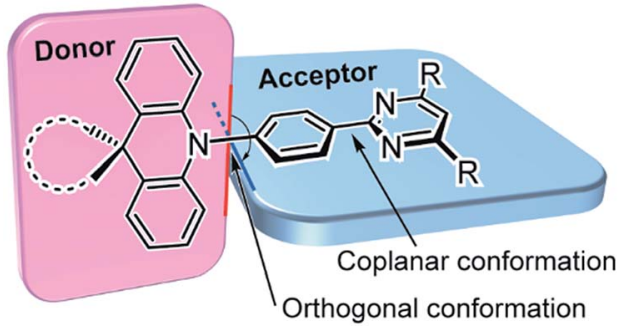

Fig. 1 Molecular design and preferred geometry of deep-blue TADF emitters based on pre-twisted acridan-pyrimidine D-A structures. phenylene ring $\left(\theta_{2}\right)$ were rather small $\left(<6^{\circ}\right)$. Such nearly orthogonal molecular structures formed by 1-5 can effectively break the $\pi$-conjugation between the donor and acceptor moieties and cause localization of the HOMO and LUMO primarily on the acridan and PPM (or PM) units, respectively. Besides, the calculated first excited $S_{1}$ states for 1-5 were dominated by the HOMO $\rightarrow$ LUMO intramolecular chargetransfer (ICT) transition. As a result, small $\Delta E_{\mathrm{ST}}$ values in the range of $0.13-0.18 \mathrm{eV}$ were estimated for 1-5 from the calculated $\mathrm{S}_{1}$ and $\mathrm{T}_{1}$ energies (Fig. 2), allowing for efficient RISC and consequently resulting in TADF emission.

The configuration of $\mathbf{1}$ was further verified using X-ray crystallographic analysis (Fig. 3). As per our design, 1 revealed a highly twisted molecular structure with a dihedral angle between the acridan unit and the adjacent phenylene ring of $80^{\circ}$, reasonably consistent with the TD-DFT calculations. It is also noted that the spiro-fused fluorene substituent caused a slight bending of the acridan unit along the C9-N10 axis, on account of the $\mathrm{sp}^{3}$ character of the $\mathrm{C} 9$ atom.

Compounds 1-5 were readily synthesized in high yields of over $90 \%$ through the Buchwald-Hartwig amination of bromoPPM (for 1 and 2) or bromo-PM (for 3-5) with the corresponding spiroacridan/acridan by employing a $\mathrm{Pd}(\mathrm{OAc})_{2} / \mathrm{P}(t-\mathrm{Bu})_{3} \mathrm{HBF}_{4}$ catalytic system. All final products were purified using temperature-gradient vacuum sublimation to obtain highly pure materials for subsequent measurements and device fabrication. The chemical structures of $\mathbf{1 - 5}$ were confirmed using ${ }^{1} \mathrm{H}$ and ${ }^{13} \mathrm{C}$ NMR spectroscopy, mass spectrometry and elemental analysis. The detailed synthetic procedures and characterization data are described in the Experimental section and ESI. $\uparrow$ The thermal properties of 1-5 were examined using thermogravimetric analysis (ESI $\dagger$ ). Among these new compounds, 1 and 2 possessed the highest thermal stability with a decomposition temperature ( $T_{\mathrm{d}}$, corresponding to $5 \%$ weight loss) of $422{ }^{\circ} \mathrm{C}$. Such a $T_{\mathrm{d}}$ value was much higher than those of 3-5 $\left(T_{\mathrm{d}}=351\right.$, 354 , and $288^{\circ} \mathrm{C}$, respectively). The $\mathrm{D}-\mathrm{A}$ molecules bearing the spiro-fused D units (MFAC and MXAc) were found to exhibit better thermal properties than that with the non-spiro Ac unit.

\section{Photophysical and TADF properties}

The steady-state UV-vis absorption and photoluminescence (PL) spectra of 1-5 in dilute solution are depicted in Fig. 4 and their relevant photophysical data are summarized in Table 1. All these compounds exhibit similar spectral features which involve two major absorption bands. While the stronger higherenergy absorptions below $330 \mathrm{~nm}$ are attributed to the $\pi-\pi^{*}$ transitions of the conjugated aromatic units, the much weaker lower-energy absorptions spanning the range of 350-400 $\mathrm{nm}$ are assigned to the ICT transitions associated with electron transfer from the acridan to the pyrimidine moieties. Upon photoexcitation at the ICT absorption band, 1-5 in toluene solution exhibited intense deep-blue PL emission with peaks $\left(\lambda_{\mathrm{PL}}\right)$ ranging from 448 to $460 \mathrm{~nm}$.

The photophysical and TADF properties of 1-5 were examined using doped thin films in a solid host matrix. The $S_{1}$ and $T_{1}$ energies ( $E_{\mathrm{S}}$ and $E_{\mathrm{T}}$, respectively) of 1-5 were determined from 


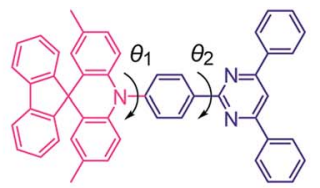

MFAc-PPM (1)

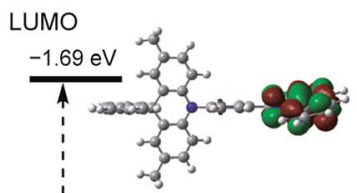

$$
E_{\mathrm{g}}=3.24 \mathrm{eV} \quad \mathrm{S}_{1} \frac{2.54 \mathrm{eV}}{\uparrow}
$$

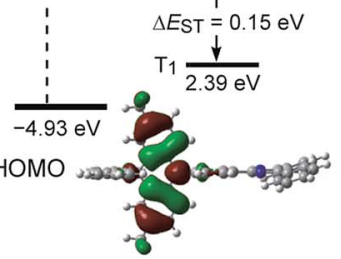

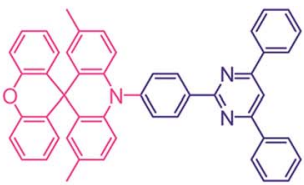

MXAc-PPM (2)

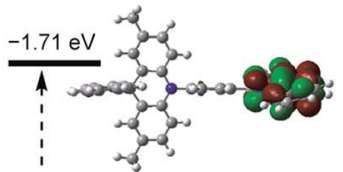

$E_{\mathrm{g}}=3.28 \mathrm{eV} \quad \mathrm{S}_{1} \frac{2.59 \mathrm{eV}}{\uparrow}$

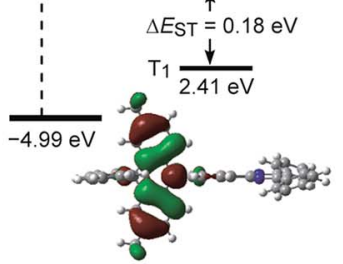

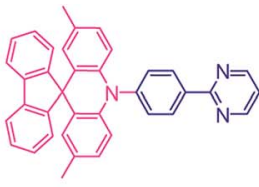

MFAc-PM (3)

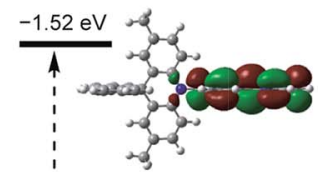

$\begin{array}{cl}E_{\mathrm{g}}=3.44 \mathrm{eV} & \mathrm{S}_{1} \frac{2.51 \mathrm{eV}}{\uparrow} \\ \vdots & \Delta E_{\mathrm{ST}}=0.13 \mathrm{eV}\end{array}$

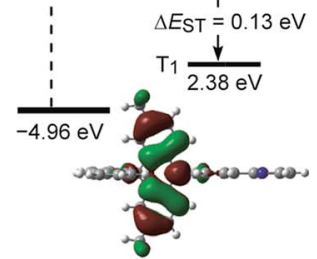

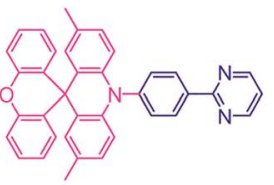

MXAc-PM (4)

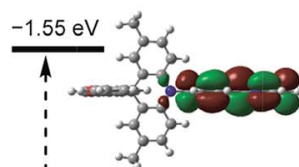

$E_{g}=3.47 \mathrm{eV} \mathrm{S} 1 \stackrel{2.54 \mathrm{eV}}{ }$

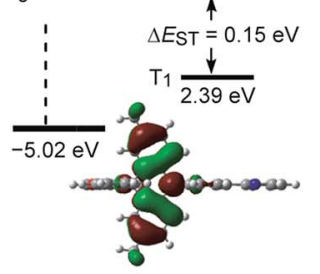

Ac-PM (5)

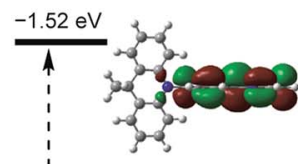

$E_{\mathrm{g}}=3.54 \mathrm{eV} \quad \mathrm{S}_{1} \underline{2.60 \mathrm{eV}}$

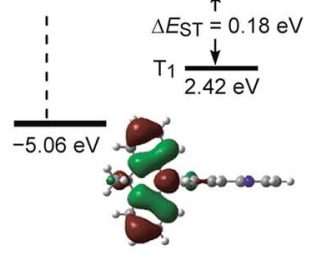

Fig. 2 Chemical structures (upper), HOMO and LUMO distributions, and calculated singlet $\left(\mathrm{S}_{1}\right)$ and triplet $\left(\mathrm{T}_{1}\right)$ energy levels (lower) for $\mathrm{D}-\mathrm{A}$ molecules 1-5 characterized using TD-DFT at the PBE1PBE/6-31G(d) level.

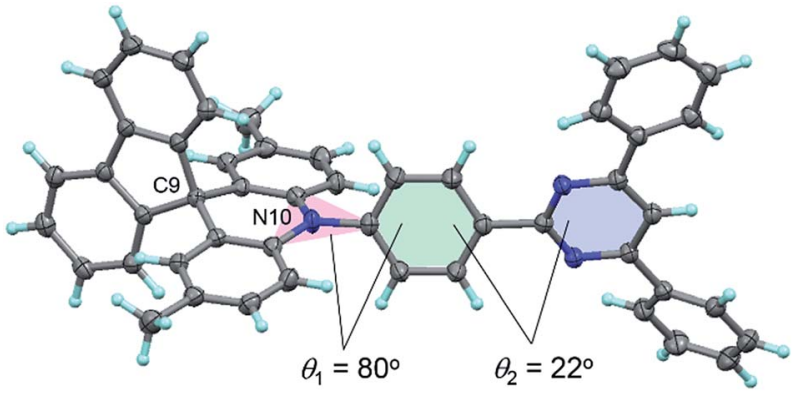

Fig. 3 ORTEP diagram of 1 with $50 \%$ probability ellipsoids. Atom color code: $\mathrm{C}$, gray; $\mathrm{N}$, blue; $\mathrm{H}$, light-blue. (a)

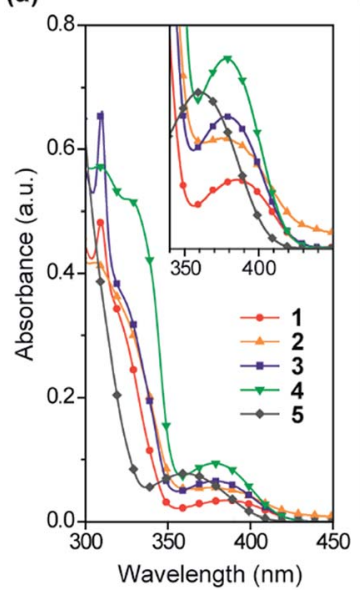

(b)

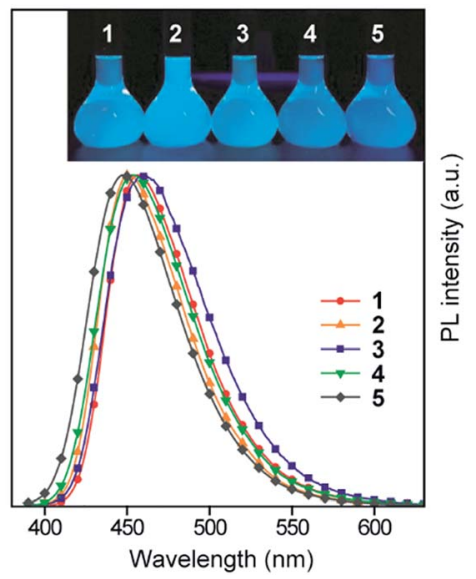

Fig. 4 (a) UV-vis absorption and (b) PL spectra of $1-5$ in toluene $\left(10^{-5}\right.$ $M)$. The insets of (a) and (b) represent a magnified view of the lowerenergy ICT absorptions and a photograph of the deep-blue PL emission from their solutions under UV irradiation, respectively. the onset of the fluorescence (300 K) and phosphorescence (5 K) spectra, respectively, and thus their $\Delta E_{\mathrm{ST}}$ values were experimentally evaluated to be between $0.25-0.30 \mathrm{eV}$ (Table 1 and ESI $\dagger$ ). Because of the high $E_{\mathrm{S}}$ and $E_{\mathrm{T}}$ values of $3.0-3.1 \mathrm{eV}$ and 2.8-2.9 eV, respectively, for these wide-bandgap emitters 1-5, we selected 2,8-bis(diphenylphosphoryl)dibenzo[ $b, d]$ furan $(\mathrm{PPF})^{39}$ with a high $E_{\mathrm{T}}$ of $3.1 \mathrm{eV}$ as a suitable host material to prevent the reverse energy transfer from the $\mathrm{T}_{1}$ states of the guest emitter to the host material and to confine the excitons in the emitters. As shown in Fig. 5, the PL emission from these doped films thoroughly originated from their guest emitters (15), manifesting an efficient host-to-guest energy transfer. Among these derivatives, MFAc-containing $\mathbf{1}$ and $\mathbf{3}$ showed slightly red-shifted PL emissions centered at 464 and $466 \mathrm{~nm}$, respectively, compared with their MXAc-containing counterparts $\left(\lambda_{\mathrm{PL}}=452\right.$ and $458 \mathrm{~nm}$ for 2 and 4 , respectively), presumably because of the enhanced electron-donating effects caused by the conjugated spirofluorene substituent on the C9 position of the acridan unit. The absolute PL quantum yields $\left(\Phi_{\mathrm{PL}}\right)$ of the doped films of 1-5 in PPF are as high as $87 \%, 69 \%$, $91 \%, 90 \%$, and $83 \%$ under $\mathrm{N}_{2}$, respectively, which are much higher than those obtained in dilute solutions $\left(\Phi_{\mathrm{PL}}=32-36 \%\right.$ in deoxygenated toluene solution). Such a PL enhancement in the solid state originates from the suppression of the non-radiative deactivation processes caused by collisional and intramolecular rotational excited-energy loss. It is noteworthy that most of these derivatives exhibited $\mathrm{CIE}_{x, y}$ values below 0.15 in those solid thin films, demonstrating their suitability as efficient deep-blue emitters in TADF-OLEDs.

The TADF characteristics of 1-5 in the doped films were further evidenced by investigating the temperature-dependent transient PL decay. As shown in Fig. 6, each of the transient PL curves displays a clear double-exponential decay profile with prompt and delayed components in oxygen-free conditions. 
Table 1 Photophysical data for deep-blue TADF emitters 1-5

\begin{tabular}{|c|c|c|c|c|c|c|c|c|c|c|c|c|}
\hline & $\lambda_{\mathrm{abs}^{a}}{ }^{a}(\mathrm{~nm})$ & $\lambda_{\mathrm{PL}}{ }^{a}(\mathrm{~nm})$ & $\lambda_{\mathrm{PL}}^{b}(\mathrm{~nm})$ & $\operatorname{CIE}^{b, c}(x, y)$ & $\Phi_{\mathrm{PL}}^{b, d}(\%)$ & $\tau_{\mathrm{p}}^{e}(\mathrm{~ns})$ & $\tau_{\mathrm{d}}^{e}(\mu \mathrm{s})$ & $\operatorname{HOMO}^{f}(\mathrm{eV})$ & $\operatorname{LUMO}^{g}(\mathrm{eV})$ & $E_{\mathrm{S}}{ }^{h}(\mathrm{eV})$ & $E_{\mathrm{T}}^{h}(\mathrm{eV})$ & $\Delta E_{\mathrm{ST}}^{i}(\mathrm{eV})$ \\
\hline 1 & 309,386 & 458 & 464 & $(0.15,0.15)$ & 87 & 12 & 38 & -5.62 & -2.67 & 3.07 & 2.82 & 0.25 \\
\hline 2 & 306,378 & 451 & 452 & $(0.15,0.12)$ & 69 & 11 & 40 & -5.65 & -2.68 & 3.10 & 2.85 & 0.25 \\
\hline 4 & 310,379 & 454 & 458 & $(0.15,0.13)$ & 90 & 11 & 70 & -5.65 & -2.70 & 3.09 & 2.80 & 0.29 \\
\hline 5 & 286,359 & 448 & 457 & $(0.15,0.13)$ & 83 & 11 & 78 & -5.68 & -2.70 & 3.10 & 2.80 & 0.30 \\
\hline
\end{tabular}

${ }^{a}$ Measured in toluene solution $\left(10^{-5} \mathrm{M}\right)$ at room temperature. ${ }^{b}$ Measured in $18 \mathrm{wt} \%$-doped thin films in a PPF solid host matrix at room temperature. ${ }^{c}$ Commission Internationale de l'Éclairage (CIE) color coordinates. ${ }^{d}$ Absolute PL quantum yield evaluated using an integrating sphere under $\mathrm{N}_{2} .{ }^{e}$ PL lifetimes of the prompt $\left(\tau_{\mathrm{p}}\right)$ and delayed $\left(\tau_{\mathrm{d}}\right)$ decay components for the 18 wt\%-doped films measured at room temperature. ${ }^{f}$ Determined using photoelectron yield spectroscopy in neat films. ${ }^{g}$ LUMO $=$ HOMO $+E_{\mathrm{g}}$, in which the optical energy gap $\left(E_{\mathrm{g}}\right)$ was derived from the absorption onset of the neat film. ${ }^{h}$ Lowest singlet $\left(E_{\mathrm{S}}\right)$ and triplet $\left(E_{\mathrm{T}}\right)$ energies estimated from the onset wavelengths of the PL spectra at 300 and $5 \mathrm{~K}$ in the doped films, respectively. ${ }^{i}$ Singlet-triplet energy splitting determined experimentally using $\Delta E_{\mathrm{ST}}=E_{\mathrm{S}}-E_{\mathrm{T}}$.

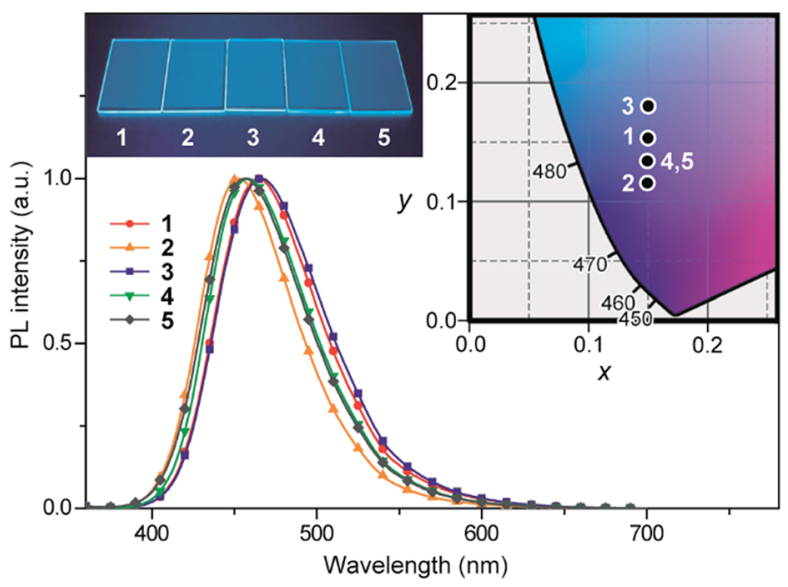

Fig. 5 PL spectra of 1-5 in 18 wt\%-emitter:PPF doped thin films. The inset shows the $\mathrm{CIE}$ chromaticity coordinates and a photograph of the deep-blue PL emission of 1-5 in the doped films.

While the prompt component with a lifetime $\left(\tau_{\mathrm{p}}\right)$ of 11-13 ns corresponds to conventional fluorescence $\left(\mathrm{S}_{1} \rightarrow \mathrm{S}_{0}\right)$, the delayed component with a lifetime $\left(\tau_{\mathrm{d}}\right)$ of 38-78 $\mu$ s can be assigned to TADF involving ISC and RISC processes $\left(\mathrm{S}_{1} \rightarrow \mathrm{T}_{1} \rightarrow \mathrm{S}_{1} \rightarrow \mathrm{S}_{0}\right)$. In comparison with $\mathbf{4}$ and $\mathbf{5}$, the relatively shorter $\tau_{\mathrm{d}}$ for $\mathbf{1 - 3}$ can be attributed to their smaller $\Delta E_{\mathrm{ST}}$ values (Table 1). Furthermore, the transient PL profiles of the doped films reveal a typical TADF feature: ${ }^{8}$ the PL intensity for the delayed component gradually increases when increasing the temperature from 5 to $300 \mathrm{~K}$. These observations unambiguously indicate that 1-5 can indeed utilize $T_{1}$ excitons for efficient light emission from the $S_{1}$ state via the $\mathrm{T}_{1} \rightarrow \mathrm{S}_{1}$ thermal upconversion. From the overall $\Phi_{\mathrm{PL}}$ value and the proportion of the integrated areas of the two components in each transient PL curve, the fractional quantum efficiencies for the prompt $\left(\Phi_{\mathrm{p}}\right)$ and delayed $\left(\Phi_{\mathrm{d}}\right)$ components were evaluated for the doped films of $\mathbf{1 - 5}$, as given in the insets of Fig. 6. Obviously, these doped films exhibited a high ratio of $\Phi_{\mathrm{d}}$ with respect to the overall $\Phi_{\mathrm{PL}}$ at ambient temperature (300 $\mathrm{K}$ ), suggesting that a large portion of the $\mathrm{S}_{1}$ excitons underwent efficient ISC and RISC and then decayed to emit delayed fluorescence upon photoexcitation. Indeed, for 1-5, high RISC efficiencies $\left(\Phi_{\text {RISC }}\right)$ of $44-82 \%$ were assumed by the equation: ${ }^{22}$ $\Phi_{\mathrm{RISC}}=\Phi_{\mathrm{d}} /\left(1-\Phi_{\mathrm{p}}\right)$ (see the ESI for details $\left.\dagger\right)$.

\section{Electroluminescence performance}

To investigate the EL performance of deep-blue TADF emitters 1-5, multilayer OLEDs were fabricated by employing thin films of 1-5 doped in a PPF host as an emitting layer (EML). We adopted the following device configuration: indium-tin-oxide (ITO, $100 \mathrm{~nm}) /$ HAT-CN $(10 \mathrm{~nm}) / \alpha$-NPD $(40 \mathrm{~nm}) / \mathrm{CCP}(5 \mathrm{~nm}) /$ EML $(20 \mathrm{~nm}) / \mathrm{PPF}(10 \mathrm{~nm}) / \mathrm{TPBi}(30 \mathrm{~nm}) / \mathrm{Liq}(1 \mathrm{~nm}) / \mathrm{Al}(100 \mathrm{~nm})$, as illustrated in Fig. 7a. In this device architecture, HAT-CN (2,3,6,7,10,11-hexacyano-1,4,5,8,9,12-hexaazatriphenylene) and $\alpha$-NPD (4,4'-bis-[ $N$-(1-naphthyl)- $N$-phenylamino]-1,1'-biphenyl) were used as a hole-injection layer and a hole-transporting layer, respectively; whereas, TPBi (1,3,5-tris $(N$-phenylbenzimidazol-2-yl)benzene) and Liq (8-hydroxyquinoline lithium) served as an electron-transporting layer and an electron-injection material, respectively. Additionally, thin layers of $\mathrm{CCP}^{33}$ (9-phenyl-3,9'-bicarbazole) and $\mathrm{PPF}^{39}$ with a high $E_{\mathrm{T}}$ of 3.0 (a)

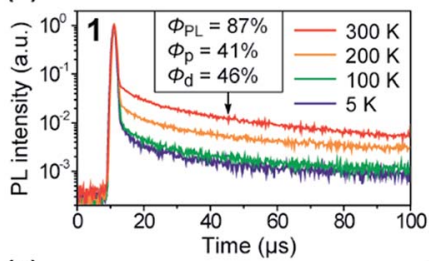

(c)

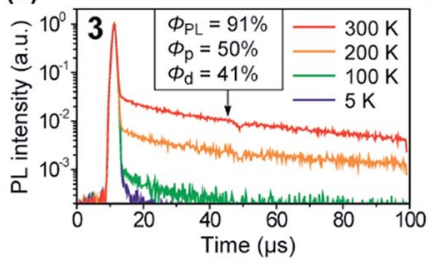

(e)

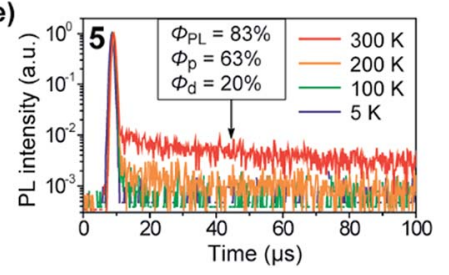

(b)

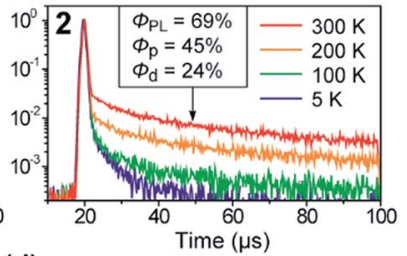

(d)

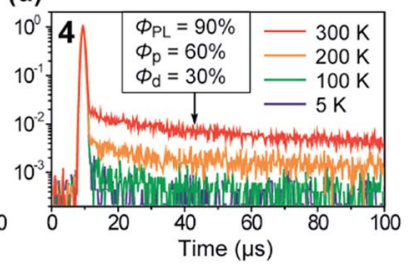

Fig. 6 Temperature dependence of the transient PL decay for 1-5 in $18 \mathrm{wt} \%$-emitter:PPF doped thin films in the temperature range of 5$300 \mathrm{~K}$ under vacuum. 
(a)

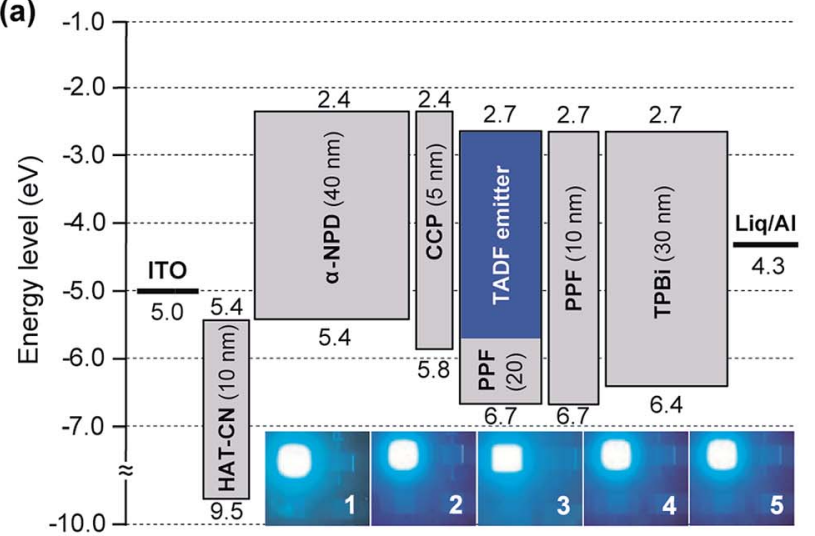

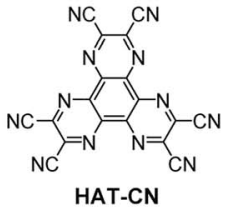

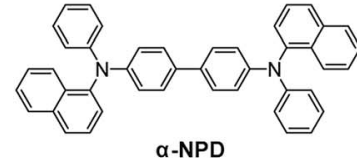

$\alpha-N P D$

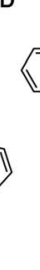

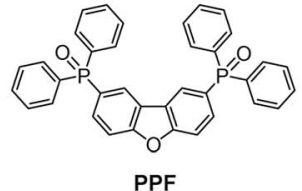

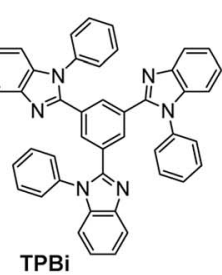

(b)

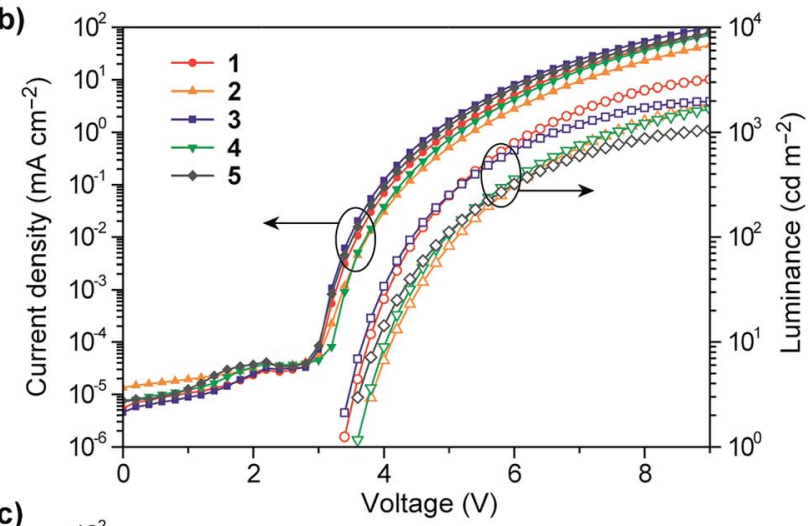

(c)

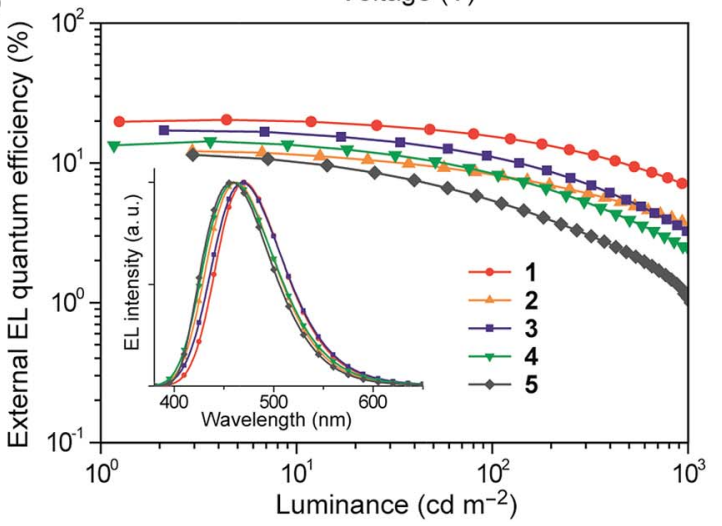

Fig. 7 (a) Schematic energy-level diagram and photos of the EL emission for the blue TADF-OLEDs based on 1-5 as emitters (top) and chemical structures of the materials used in the devices (bottom). (b) Current density and luminance versus voltage ( $J-V-L$ ) characteristics and (c) external EL quantum efficiency versus luminance $\left(\eta_{\text {ext }}-L\right)$ characteristics of the blue TADF-OLEDs. The inset of (c) represents the EL spectra measured at $10 \mathrm{~mA} \mathrm{~cm}^{-2}$.

and $3.1 \mathrm{eV}$ were inserted as exciton-blocking layers to suppress the triplet exciton deactivation at the neighboring interfaces and to confine the excitons within the EML.

The EL characteristics of the fabricated TADF-OLEDs are depicted in Fig. $7 \mathrm{~b}$ and c, and the key device parameters are compiled in Table 2. The devices based on 1-5 displayed bright blue EL emission peaking in the range of $458-470 \mathrm{~nm}$, with rather low turn-on voltages $\left(V_{\text {on }}\right)$ of 3.4-3.6 V. Their EL spectra

Table 2 EL performance of the TADF-OLEDs based on 1-5

\begin{tabular}{llllll}
\hline Emitter $^{a}$ & $\mathbf{1}$ & $\mathbf{2}$ & $\mathbf{3}$ & $\mathbf{4}$ & $\mathbf{5}$ \\
\hline$\lambda_{\mathrm{EL}}{ }^{b}(\mathrm{~nm})$ & 470 & 462 & 469 & 460 & 458 \\
$V_{\mathrm{on}}{ }^{c}(\mathrm{~V})$ & 3.4 & 3.6 & 3.4 & 3.6 & 3.6 \\
$\eta_{\text {ext,max }}{ }^{c}(\%)$ & 20.4 & 12.2 & 17.1 & 14.3 & 11.4 \\
$\eta_{\text {ext, } 100}{ }^{e}(\%)$ & 15.6 & 8.2 & 10.9 & 8.4 & 5.4 \\
$\eta_{\mathrm{c}}{ }^{f}\left(\mathrm{~cd} \mathrm{~A}^{-1}\right)$ & 41.7 & 22.7 & 34.3 & 25.0 & 18.9 \\
$\eta_{\mathrm{p}}{ }^{g}\left(\operatorname{lm~W}{ }^{-1}\right)$ & 37.2 & 18.8 & 31.7 & 20.7 & 16.5 \\
$\mathrm{CIE}^{h}(x, y)$ & $(0.16$, & $(0.16$, & $(0.16$, & $(0.16$, & $(0.15$, \\
& $0.23)$ & $0.20)$ & $0.21)$ & $0.19)$ & $0.15)$
\end{tabular}

${ }^{a}$ Device configuration: ITO/HAT-CN $(10 \mathrm{~nm}) / \alpha-\mathrm{NPD}(40 \mathrm{~nm}) / \mathrm{CCP}$ $(5 \mathrm{~nm}) / 18$ wt\%-emitter:PPF $(20 \mathrm{~nm}) / \mathrm{PPF}(10 \mathrm{~nm}) / \mathrm{TPBi}(30 \mathrm{~nm}) / \mathrm{Liq}$ $(1 \mathrm{~nm}) / \mathrm{Al}(100 \mathrm{~nm}) .{ }^{b}$ EL emission maximum. ${ }^{c}$ Turn-on voltage at a brightness of $1 \mathrm{~cd} \mathrm{~m}^{-2} \cdot{ }^{d}$ Maximum external EL quantum efficiency. ${ }^{e}$ External EL quantum efficiency at $100 \mathrm{~cd} \mathrm{~m}^{-2} .{ }^{f}$ Maximum current efficiency. ${ }^{g}$ Maximum power efficiency. ${ }^{h}$ Commission Internationale de l'Éclairage (CIE) chromaticity coordinates recorded at $10 \mathrm{~mA} \mathrm{~cm}{ }^{-2}$. were consistent with the corresponding PL spectra, suggesting efficient carrier injection, transport, and recombination into the EML within the device. Among the fabricated devices, the device employing 1 achieved the highest EL efficiencies with a maximum $\eta_{\text {ext }}$ of $20.4 \%$, current efficiency $\left(\eta_{\mathrm{c}}\right)$ of $41.7 \mathrm{~cd} \mathrm{~A}^{-1}$, and power efficiency $\left(\eta_{\mathrm{p}}\right)$ of $37.2 \mathrm{~lm} \mathrm{~W}^{-1}$ at low current densities without any light out-coupling enhancement. The CIE coordinates of the EL from this device were $(0.16,0.23)$. To our knowledge, these efficiencies are among the highest level for blue TADF-OLEDs ever reported..$^{10,16,21,22,29,33,34,38}$ So far, deepblue TADF-OLEDs with emission maxima $\left(\lambda_{\mathrm{EL}}\right)$ below $470 \mathrm{~nm}$ have rarely achieved a high $\eta_{\text {ext }}$ exceeding $20 \%$. Moreover, the device employing 1 also showed a relatively reduced efficiency roll-off compared to the other devices; the $\eta_{\text {ext }}$ value still remained as high as $15.6 \%$ at a practical luminance of $100 \mathrm{~cd}$ $\mathrm{m}^{-2}$. The reduced roll-off behavior for $\mathbf{1}$ can be attributed to the fast RISC originating from its relatively shorter $\tau_{\mathrm{d}}$ and the suppression of triplet-triplet annihilation (TTA) and singlettriplet annihilation (STA), ${ }^{\mathbf{4 0 , 4}}$ as discussed below.

Comparing the performance of the TADF-OLEDs containing 1-5, the maximum $\eta_{\text {ext }}$ values were in the order of $\mathbf{1}(20.4 \%)>3$ $(17.1 \%)>4(14.3 \%)>2(12.2 \%)>5(11.4 \%)$. The relatively lower efficiencies of the devices with 2 and 5 compared to those with 1,3 , and 4 can be mainly ascribed to their lower $\Phi_{\mathrm{PL}}$ and $\Phi_{\mathrm{d}}$ values. Nevertheless, the $\eta_{\text {ext }}$ values of $\mathbf{2}$ and $\mathbf{5}$ were more than two times higher than those expected from conventional 
fluorescent emitters with the same $\Phi_{\mathrm{PL}}$ values. These pyrimidine-based deep-blue TADF emitters could thus achieve high EL efficiencies by utilizing both the electro-generated $T_{1}$ and $S_{1}$ excitons for efficient light emission. However, the EL efficiencies for some of these TADF-OLEDs significantly decreased with increasing current density (or luminance). This severe efficiency roll-off is primarily attributed to the long-lived excited states of the $\mathrm{T}_{1}$ excitons, which undergo exciton deactivation processes such as TTA and STA. The TTA model is used here to analyze the efficiency roll-off for the devices containing 1-5, according to the following equation: ${ }^{\mathbf{4 0 - 4 2}}$

$$
\eta_{\mathrm{ext}} / \eta_{0}=J / 4 J_{0}\left[\sqrt{1+8 J / J_{0}}-1\right]
$$

where $\eta_{0}$ is the external EL quantum efficiency in the absence of TTA and $J_{0}$ is the critical current density at $\eta_{\text {ext }}=\eta_{0} / 2$. The fitted curves based on the TTA model agreed well with the experimental $\eta_{\text {ext }}-J$ plots for all the devices containing 1-5 with correlation coefficients greater than 0.98 (ESI $\dagger$ ), which indicates that the efficiency roll-off for these devices was primarily caused by TTA exciton deactivation. Indeed, the device based on 5 showed a smaller $J_{0}$ value $\left(0.9 \mathrm{~mA} \mathrm{~cm}{ }^{-2}\right)$ than that of $1\left(2.1 \mathrm{~mA} \mathrm{~cm}{ }^{-2}\right)$, which implies that $\mathbf{5}$ suffered from more severe TTA and efficiency roll-off as the current density increased. This propensity arises from the relatively long TADF lifetime $\left(\tau_{\mathrm{d}}\right)$ of 5 in the doped film. If efficient deep-blue TADF emitters with a much shorter $\tau_{\mathrm{d}}(<1 \mu \mathrm{s})$ can be realized, we can therefore expect that high $\eta_{\text {ext }}$ values of over $20 \%$ can be retained even at higher current densities.

\section{Conclusions}

A new family of deep-blue TADF emitters, consisting of pretwisted acridan-pyrimidine D-A motifs, were designed and synthesized. All of these emitters in doped thin films showed excellent PL properties with quantum yields of $69-91 \%$ accompanied by prominent TADF originating from their small
$\Delta E_{\mathrm{ST}}$. By employing these TADF emitters for OLEDs, considerably high maximum external EL quantum efficiencies of up to $20.4 \%$ with CIE coordinates of $(0.16,0.23)$ were achieved. Deepblue EL with CIE coordinates of $(0.15,0.15)$ could also be obtained through rational molecular design in this platform. These results validate a versatile design strategy to utilize pyrimidine derivatives as a universal platform for the further development of efficient deep-blue organic emitters.

\section{Experimental section}

\section{Materials and synthesis}

All commercially available reagents and solvents were used as received unless otherwise noted. 2,8-Bis(diphenylphosphoryl) dibenzo[ $b, d]$ furan $(\mathrm{PPF})^{39}$ and 9-phenyl-3,9'-bicarbazole (CCP) ${ }^{33}$ were prepared according to the literature procedures, and were purified using vacuum sublimation. 2,3,6,7,10,11-Hexacyano1,4,5,8,9,12-hexaazatriphenylene (HAT-CN) was donated by Nippon Soda Co., Ltd. and was purified using vacuum sublimation before use. Other OLED materials were purchased from E-Ray Optoelectronics Technology Co., Ltd. and were used for the device fabrication without further purification. The synthesis routes for deep-blue TADF molecules 1-5 are outlined in Scheme 1, and detailed synthetic procedures and characterization data for other intermediates (6-9) are given in the ESI. $\dagger$ 9,9Dimethylacridan $^{43}(\mathbf{1 0})$ was prepared according to the literature procedure. All final products were purified using temperaturegradient vacuum sublimation with a P-100 system (ALS Technology), before the measurements and device fabrication.

MFAc-PPM (1). A mixture of $6(1.00 \mathrm{~g}, 2.58 \mathrm{mmol}), 8(0.93 \mathrm{~g}$, $2.59 \mathrm{mmol}), \mathrm{Pd}(\mathrm{OAc})_{2}(0.022 \mathrm{~g}, 0.1 \mathrm{mmol}), \mathrm{P}(t-\mathrm{Bu})_{3} \mathrm{HBF}_{4}(0.03 \mathrm{~g}$, $0.1 \mathrm{mmol})$, and $\mathrm{K}_{2} \mathrm{CO}_{3}(1.07 \mathrm{~g}, 7.7 \mathrm{mmol})$ in dry toluene $(30 \mathrm{~mL})$ was refluxed for $48 \mathrm{~h}$ under $\mathrm{N}_{2}$. After cooling to room temperature, the reaction mixture was filtered through a Celite pad and then the filtrate was concentrated under reduced pressure. The product was purified using column chromatography on silica gel (eluent:

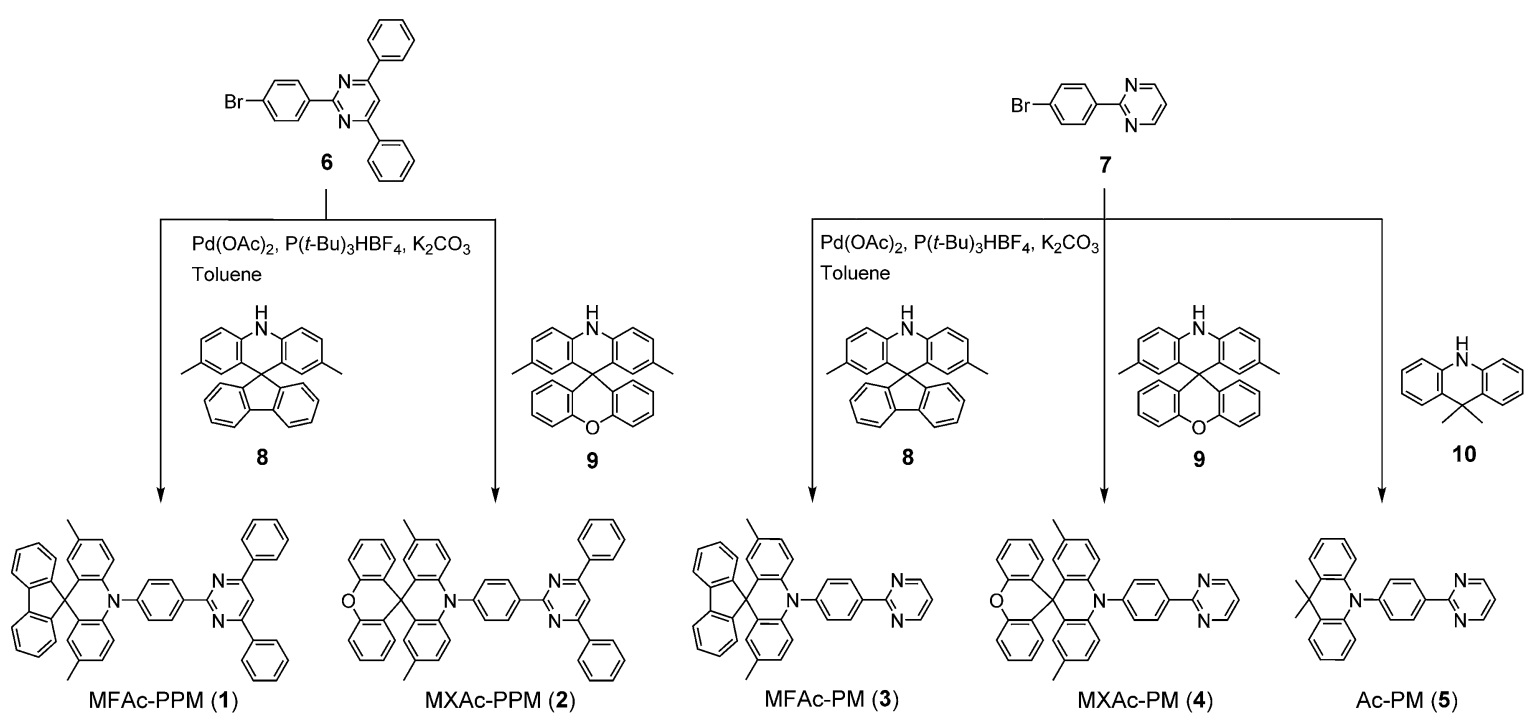

Scheme 1 Synthesis routes for pyrimidine-based TADF molecules 1-5. 
hexane/chloroform $=3: 1, \mathrm{v} / \mathrm{v}$ ) to afford 1 as a white solid (yield $=$ $1.60 \mathrm{~g}, 93 \%) .{ }^{1} \mathrm{H}$ NMR (400 MHz, DMSO- $d_{6}$ ): $\delta 9.03$ (d, $J=8.4 \mathrm{~Hz}$, $2 \mathrm{H}), 8.67(\mathrm{~s}, 1 \mathrm{H}), 8.60-8.58(\mathrm{~m}, 4 \mathrm{H}), 7.99(\mathrm{~d}, J=7.6 \mathrm{~Hz}, 2 \mathrm{H}), 7.78$ $(\mathrm{d}, J=8.4 \mathrm{~Hz}, 2 \mathrm{H}), 7.68-7.66(\mathrm{~m}, 6 \mathrm{H}), 7.45(\mathrm{td}, J=7.4 \mathrm{~Hz}, 1.3 \mathrm{~Hz}$, $2 \mathrm{H}), 7.41(\mathrm{~d}, J=7.2 \mathrm{~Hz}, 2 \mathrm{H}), 7.33(\mathrm{td}, J=7.2 \mathrm{~Hz}, 1.2 \mathrm{~Hz}, 2 \mathrm{H}), 6.80$ $(\mathrm{dd}, J=8.6 \mathrm{~Hz}, 1.8 \mathrm{~Hz}, 2 \mathrm{H}), 6.32(\mathrm{~d}, J=8.4 \mathrm{~Hz}, 2 \mathrm{H}), 6.04(\mathrm{~d}, J=1.6$ $\mathrm{Hz}, 2 \mathrm{H}), 1.90$ (s, 6H). ${ }^{13} \mathrm{C} \mathrm{NMR}\left(100 \mathrm{MHz}, \mathrm{CDCl}_{3}\right): \delta 165.04,164.02$, 156.52, 143.81, 139.24, 139.22, 138.16, 137.39, 131.38, 131.19, $130.98,129.48,129.03,128.40,128.00,127.87,127.45,127.34$, 125.86, 124.63, 119.83, 114.52, 110.55, 56.92, 20.40. MS (MALDITOF): $m / z$ calcd $665.28[\mathrm{M}]^{+}$; found 665.18. Anal. calcd (\%) for $\mathrm{C}_{49} \mathrm{H}_{35} \mathrm{~N}_{3}$ : C 88.39, H 5.30, N 6.31; found: C 88.35, H 5.23, N 6.34.

MXAc-PPM (2). This compound was synthesized according to the same procedure as described above for the synthesis of $\mathbf{1}$, except that $9(0.97 \mathrm{~g}, 2.58 \mathrm{mmol})$ was used as the reactant instead of 8, yielding 2 as a white solid (yield $=1.60 \mathrm{~g}, 91 \%) .{ }^{1} \mathrm{H}$ NMR (400 MHz, DMSO- $\left.d_{6}\right): \delta 9.01(\mathrm{~d}, J=8.8 \mathrm{~Hz}, 2 \mathrm{H}), 8.66(\mathrm{~s}$, $1 \mathrm{H}), 8.60-8.57(\mathrm{~m}, 4 \mathrm{H}), 7.73(\mathrm{~d}, J=8.8 \mathrm{~Hz}, 2 \mathrm{H}), 7.67-7.66(\mathrm{~m}$, $6 \mathrm{H}), 7.26(\mathrm{dd}, J=6.0 \mathrm{~Hz}, 1.6 \mathrm{~Hz}, 4 \mathrm{H}), 7.15(\mathrm{dd}, J=8.8 \mathrm{~Hz}, 1.2 \mathrm{~Hz}$, 2H), 7.08-7.04 (m, 2H), $6.76(\mathrm{dd}, J=8.6 \mathrm{~Hz}, 1.4 \mathrm{~Hz}, 2 \mathrm{H}), 6.48$ (d, $J=2.0 \mathrm{~Hz}, 2 \mathrm{H}), 6.24(\mathrm{~d}, J=8.4 \mathrm{~Hz}, 2 \mathrm{H}), 1.96(\mathrm{~s}, 6 \mathrm{H}) .{ }^{13} \mathrm{C} \mathrm{NMR}$ $\left(100 \mathrm{MHz}, \mathrm{CDCl}_{3}\right): \delta$ 165.06, 163.99, 148.44, 138.22, 137.38, $137.14,132.54,132.06,131.54,131.43,131.25,130.99,129.74$, 129.52, 129.04, 127.83, 127.45, 127.34, 123.69, 115.85, 114.06, 110.58, 44.74, 20.48. MS (MALDI-TOF): $\mathrm{m} / z$ calcd $681.28[\mathrm{M}]^{+}$; found 681.11. Anal. calcd (\%) for $\mathrm{C}_{49} \mathrm{H}_{35} \mathrm{~N}_{3} \mathrm{O}: \mathrm{C} 86.32, \mathrm{H} \mathrm{5.17,} \mathrm{N}$ 6.16; found: C 86.45, H 5.11, N 6.31.

MFAc-PM (3). A mixture of $7(1.00 \mathrm{~g}, 4.25 \mathrm{mmol}), 8(1.55 \mathrm{~g}$, $4.31 \mathrm{mmol}), \mathrm{Pd}(\mathrm{OAc})_{2}(0.03 \mathrm{~g}, 0.14 \mathrm{mmol}), \mathrm{P}(t-\mathrm{Bu})_{3} \mathrm{HBF}_{4}(0.03 \mathrm{~g}$, $0.1 \mathrm{mmol})$, and $\mathrm{K}_{2} \mathrm{CO}_{3}(1.80 \mathrm{~g}, 13.0 \mathrm{mmol})$ in dry toluene (80 $\mathrm{mL}$ ) was refluxed for $12 \mathrm{~h}$ under $\mathrm{N}_{2}$. After cooling to room temperature, the reaction mixture was filtered through a Celite pad and then the filtrate was concentrated under reduced pressure. The product was purified using column chromatography on silica gel (eluent: hexane/chloroform $=3: 1, \mathrm{v} / \mathrm{v}$ ) to afford 3 as a white solid (yield $=2.01 \mathrm{~g}, 92 \%) .{ }^{1} \mathrm{H}$ NMR (400 MHz, DMSO- $d_{6}$ ): $\delta 9.02(\mathrm{~d}, J=4.8 \mathrm{~Hz}, 2 \mathrm{H}), 8.77(\mathrm{dd}, J=6.4 \mathrm{~Hz}$, $2.0 \mathrm{~Hz}, 2 \mathrm{H}), 7.98(\mathrm{~d}, J=7.6 \mathrm{~Hz}, 2 \mathrm{H}), 7.71(\mathrm{dd}, J=6.8 \mathrm{~Hz}, 2.0 \mathrm{~Hz}$, $2 \mathrm{H}), 7.55(\mathrm{t}, J=4.8 \mathrm{~Hz}, 1 \mathrm{H}), 7.44(\mathrm{td}, J=7.2 \mathrm{~Hz}, 1.2 \mathrm{~Hz}, 2 \mathrm{H}), 7.39$ $(\mathrm{d}, J=7.2 \mathrm{~Hz}, 2 \mathrm{H}), 7.32(\mathrm{td}, J=7.2 \mathrm{~Hz}, 1.2 \mathrm{~Hz}, 2 \mathrm{H}), 6.78(\mathrm{dd}, J=$ $8.8 \mathrm{~Hz}, 1.9 \mathrm{~Hz}, 2 \mathrm{H}), 6.26$ (d, $J=8.4 \mathrm{~Hz}, 2 \mathrm{H}), 6.03(\mathrm{~d}, J=2.0 \mathrm{~Hz}$, $2 \mathrm{H}), 1.89$ (s, 6H). ${ }^{13} \mathrm{C} \mathrm{NMR}\left(100 \mathrm{MHz}, \mathrm{CDCl}_{3}\right): \delta$ 164.24, 157.44, $156.51,144.01,139.24,139.14,137.55,131.58,130.86,129.52$, 128.39, 127.98, 127.89, 127.45, 125.84, 124.61, 119.82, 119.39, 114.45, 56.88, 20.39. MS (MALDI-TOF) $m / z$ : calcd $513.22[\mathrm{M}]^{+}$; found 514.04. Anal. calcd (\%) for $\mathrm{C}_{37} \mathrm{H}_{27} \mathrm{~N}_{3}$ : C 86.52, $\mathrm{H} \mathrm{5.30,} \mathrm{N}$ 8.18; found: C 86.64, H 5.06, N 8.23.

MXAc-PM (4). This compound was synthesized according to the same procedure as described above for the synthesis of $\mathbf{3}$, except that 9 ( $1.60 \mathrm{~g}, 4.26 \mathrm{mmol})$ was used as the reactant instead of 8, yielding 4 as a white solid (yield $=2.03 \mathrm{~g}, 90 \%) .{ }^{1} \mathrm{H}$ NMR (400 MHz, DMSO- $d_{6}$ ): $\delta 9.01(\mathrm{~d}, J=5.2 \mathrm{~Hz}, 2 \mathrm{H}$ ), $8.75(\mathrm{dd}, J=6.4$ $\mathrm{Hz}, 2.0 \mathrm{~Hz}, 2 \mathrm{H}), 7.67$ (d, $J=8.4 \mathrm{~Hz}, 2 \mathrm{H}), 7.55$ (t, $J=5.0 \mathrm{~Hz}, 1 \mathrm{H})$, $7.25-7.23(\mathrm{~m}, 4 \mathrm{H}), 7.12(\mathrm{dd}, J=7.6 \mathrm{~Hz}, 1.2 \mathrm{~Hz}, 2 \mathrm{H}), 7.06-7.04(\mathrm{~m}$, $2 \mathrm{H}), 6.74(\mathrm{dd}, J=8.8 \mathrm{~Hz}, 1.9 \mathrm{~Hz}, 2 \mathrm{H}), 6.46(\mathrm{~d}, J=1.6 \mathrm{~Hz}, 2 \mathrm{H}), 6.18$ (d, $J=8.8 \mathrm{~Hz}, 2 \mathrm{H}), 1.95$ (s, 6H). ${ }^{13} \mathrm{C}$ NMR (100 MHz, $\mathrm{CDCl}_{3}$ ): $\delta 164.18,157.44,148.41,143.91,137.60,137.04,132.55,132.03$,
131.62, 131.51, 130.91, 129.77, 129.50, 127.79, 127.43, 123.66, 119.40, 115.83, 113.98, 44.70, 20.46. MS (MALDI-TOF): $\mathrm{m} / \mathrm{z}$ calcd $529.22[\mathrm{M}]^{+}$; found 529.12. Anal. calcd (\%) for $\mathrm{C}_{37} \mathrm{H}_{27} \mathrm{~N}_{3} \mathrm{O}: \mathrm{C}$ 83.91, H 5.14, N 7.93; found: C 83.84, H 5.03, N 8.02.

Ac-PM (5). This compound was synthesized according to the same procedure as described above for the synthesis of 3, except that $10(0.89 \mathrm{~g}, 4.25 \mathrm{mmol})$ was used as the reactant instead of $\mathbf{8}$, yielding 5 as a white solid (yield $=1.45 \mathrm{~g}, 94 \%) .{ }^{1} \mathrm{H}$ NMR (400 MHz, DMSO- $\left.d_{6}\right): \delta 8.99(\mathrm{~d}, J=5.2 \mathrm{~Hz}, 2 \mathrm{H}), 8.69(\mathrm{~d}, J=8.4 \mathrm{~Hz}$, 2H), 7.55-7.50 (m, 5H), $6.99(\mathrm{td}, J=7.7 \mathrm{~Hz}, 1.3 \mathrm{~Hz}, 2 \mathrm{H}), 6.93(\mathrm{td}$, $J=7.5 \mathrm{~Hz}, 1.3 \mathrm{~Hz}, 2 \mathrm{H}), 6.24(\mathrm{dd}, J=8.0 \mathrm{~Hz}, 1.2 \mathrm{~Hz}, 2 \mathrm{H}), 1.64$ (s, $6 \mathrm{H}) .{ }^{13} \mathrm{C} \mathrm{NMR}\left(100 \mathrm{MHz}, \mathrm{CDCl}_{3}\right): \delta 164.19,157.40,143.66$, $140.69,137.49,131.55,130.74,130.08,126.38,125.25,120.66$, 119.35, 114.12, 36.00, 31.28. MS (MALDI-TOF): $\mathrm{m} / \mathrm{z}$ calcd 363.17 $[\mathrm{M}]^{+}$; found 362.99. Anal. calcd (\%) for $\mathrm{C}_{25} \mathrm{H}_{21} \mathrm{~N}_{3}$ : C 82.61, H 5.82, N 11.56; found: C 82.61, H 5.75, N 11.65 .

\section{Photophysical measurements}

The UV-vis absorption and photoluminescence (PL) spectra were measured with a V-670 spectrometer (Jasco) and a FP-8600 spectrophotometer (Jasco), respectively, using degassed spectral grade solvents. The absolute PL quantum yields $\left(\Phi_{\mathrm{PL}}\right)$ were determined using an ILF-835 integrating sphere system (Jasco). The transient PL decay measurements were carried out using a C11367 Quantaurus-tau fluorescence lifetime spectrometer (Hamamatsu Photonics; $\lambda=340 \mathrm{~nm}$, pulse width $=100 \mathrm{ps}$, and repetition rate $=20 \mathrm{~Hz}$ ) under $\mathrm{N}_{2}$, and a C9300 streak camera (Hamamatsu Photonics) with an $\mathrm{N}_{2}$ gas laser $(\lambda=337 \mathrm{~nm}$, pulse width $=500 \mathrm{ps}$, and repetition rate $=20 \mathrm{~Hz})$ under vacuum $(<4$ $\times 10^{-1} \mathrm{~Pa}$ ). The HOMO energies of materials in neat films were determined using an AC-2 ultraviolet photoelectron spectrometer (Riken-Keiki). The LUMO energies were estimated by subtracting the optical energy gaps $\left(E_{\mathrm{g}}\right)$ from the measured HOMO energies; the $E_{\mathrm{g}}$ values were determined from the onset positions of the PL spectra of the thin films.

\section{OLED fabrication and characterization}

ITO-coated glass substrates were cleaned with detergent, deionized water, acetone, and isopropanol. The substrates were then subjected to UV-Ozone treatment for $30 \mathrm{~min}$ before they were loaded into an E-200 vacuum evaporation system (ALS Technology). The organic layers and a cathode aluminum layer were thermally evaporated onto the substrates under vacuum $\left(<6 \times 10^{-5} \mathrm{~Pa}\right)$ with an evaporation rate of $<0.3 \mathrm{~nm} \mathrm{~s}^{-1}$ through a shadow mask. The layer thickness and deposition rate were monitored in situ during deposition using an oscillating quartz thickness monitor. OLED characteristics were measured using a 2400 source meter (Keithley) and a CS-2000 spectroradiometer (Konica Minolta).

\section{Acknowledgements}

This work was supported in part by Grant-in-Aid for Scientific Research on Innovative Areas (No. 15H01049) from JSPS, the Cooperative Research Program of "Network Joint Research Center for Materials and Devices", the Canon Foundation, the 
Sumitomo Electric Group CSR Foundation, the Futaba Electronics Memorial Foundation, and the KDDI Foundation. I. P. acknowledges the support from the Rotary Yoneyama Scholarships. The authors thank Dr N. Aizawa for fruitful discussion regarding this work.

\section{References}

1 C. W. Tang and S. A. VanSlyke, Appl. Phys. Lett., 1987, 51, 913. 2 M. A. Baldo, S. Lamansky, P. E. Burrows, M. E. Thompson and S. R. Forrest, Appl. Phys. Lett., 1999, 75, 4.

3 C. Adachi, M. A. Baldo, M. E. Thompson and S. R. Forrest, J. Appl. Phys., 2001, 90, 5048.

4 S. Reineke, F. Lindner, G. Schwartz, N. Seidler, K. Walzer, B. Lüssem and K. Leo, Nature, 2009, 459, 234.

$5 \mathrm{H}$. Yersin, Highly Efficient OLEDs with Phosphorescent Materials, Wiley-VCH, Weinheim, Germany, 2008.

6 K. S. Yook and J. Y. Lee, Adv. Mater., 2012, 24, 3169.

7 M. Zhu and C. Yang, Chem. Soc. Rev., 2013, 42, 4963.

8 H. Uoyama, K. Goushi, K. Shizu, H. Nomura and C. Adachi, Nature, 2012, 492, 234.

9 S. Hirata, Y. Sakai, K. Masui, H. Tanaka, S. Y. Lee, H. Nomura, N. Nakamura, M. Yasumatsu, H. Nakanotani, Q. Zhang, K. Shizu, H. Miyazaki and C. Adachi, Nat. Mater., 2014, 14, 330 .

10 Q. Zhang, B. Li, S. Huang, H. Nomura, H. Tanaka and C. Adachi, Nat. Photonics, 2014, 8, 326.

11 Q. Zhang, D. Tsang, H. Kuwabara, Y. Hatae, B. Li, T. Takahashi, S. Y. Lee, T. Yasuda and C. Adachi, Adv. Mater., 2015, 27, 2096.

12 H. Kaji, H. Suzuki, T. Fukushima, K. Shizu, K. Suzuki, S. Kubo, T. Komino, H. Oiwa, F. Suzuki, A. Wakamiya, Y. Murata and C. Adachi, Nat. Commun., 2015, 6, 8476.

13 R. Komatsu, H. Sasabe, Y. Seino, K. Nakao and J. Kido, J. Mater. Chem. C, 2016, 4, 2274.

14 K. Wu, T. Zhang, L. Zhan, C. Zhong, S. Gong, N. Jiang, Z.-H. Lu and C. Yang, Chem.-Eur. J., 2016, 22, 10860.

15 M. Kim, S. K. Jeon, S.-H. Hwang and J. Y. Lee, Adv. Mater., 2015, 27, 2515.

16 J. W. Sun, J. Y. Baek, K.-H. Kim, C.-K. Moon, J.-H. Lee, S.-K. Kwon, Y.-H. Kim and J.-J. Kim, Chem. Mater., 2015, 27,6675 .

17 T.-A. Lin, T. Chatterjee, W.-L. Tsai, W.-K. Lee, M.-J. Wu, M. Jiao, K.-C. Pan, C.-L. Yi, C.-L. Chung, K.-T. Wong and C.-C. Wu, Adv. Mater., 2016, 28, 6976.

18 Q. Zhang, J. Li, K. Shizu, S. Huang, S. Hirata, H. Miyazaki and C. Adachi, J. Am. Chem. Soc., 2012, 134, 14706.

19 S. Wu, M. Aonuma, Q. Zhang, S. Huang, T. Nakagawa, K. Kuwabara and C. Adachi, J. Mater. Chem. C, 2014, 2, 421.

20 M. Liu, Y. Seino, D. Chen, S. Inomata, S.-J. Su, H. Sasabe and J. Kido, Chem. Commun., 2015, 51, 16353.
21 I. Lee and J. Y. Lee, Org. Electron., 2016, 29, 160.

22 S. Y. Lee, C. Adachi and T. Yasuda, Adv. Mater., 2016, 28, 4626.

23 S. Y. Lee, T. Yasuda, Y. S. Yang, Q. Zhang and C. Adachi, Angew. Chem., Int. Ed., 2014, 53, 6402.

24 P. Rajamalli, N. Senthilkumar, P. Gandeepan, C.-Z. Ren-Wu, H.-W. Lin and C.-H. Cheng, J. Mater. Chem. C, 2016, 4, 900.

25 P. Rajamalli, N. Senthilkumar, P. Gandeepan, P.-Y. Huang, M.-J. Huang, C.-Z. Ren-Wu, C.-Y. Yang, M.-J. Chiu, L.-K. Chu, H.-W. Lin and C.-H. Cheng, J. Am. Chem. Soc., 2016, 138, 628.

26 Z. Wang, Y. Li, X. Cai, D. Chen, G. Xie, K. Liu, Y.-C. Wu, C.-C. Lo, A. Lien, Y. Cao and S.-J. Su, ACS Appl. Mater. Interfaces, 2016, 8, 8627.

27 I. S. Park, S. Y. Lee, C. Adachi and T. Yasuda, Adv. Funct. Mater., 2016, 26, 1813.

28 Y. J. Cho, K. S. Yook and J. Y. Lee, Sci. Rep., 2015, 5, 7859.

29 D. Zhang, M. Cai, Y. Zhang, D. Zhang and L. Duan, Mater. Horiz., 2016, 3, 145.

30 D. Zhang, M. Cai, Z. Bin, Y. Zhang, D. Zhang and L. Duan, Chem. Sci., 2016, 7, 3355.

31 Y. J. Cho, S. K. Jeon, B. D. Chin, E. Yu and J. Y. Lee, Angew. Chem., Int. Ed., 2015, 54, 5201.

32 W. Liu, C.-J. Zhang, K. Wang, Z. Chen, D.-Y. Chen, F. Li, X.-M. Ou, Y.-P. Dong and X.-H. Zhang, ACS Appl. Mater. Interfaces, 2015, 7, 18930.

33 M. Numata, T. Yasuda and C. Adachi, Chem. Commun., 2015, 51, 9443.

34 T. Hatakeyama, K. Shiren, K. Nakajima, S. Nomura, S. Nakatsuka, K. Kinoshita, J. Ni, Y. Ono and T. Ikuta, Adv. Mater., 2016, 28, 2777.

35 I. S. Park, M. Numata, C. Adachi and T. Yasuda, Bull. Chem. Soc. Jpn., 2016, 89, 375.

36 Y. Kitamoto, T. Namikawa, D. Ikemizu, Y. Miyata, T. Suzuki, H. Kita, T. Sato and S. Oi, J. Mater. Chem. C, 2015, 3, 9122.

37 K. Suzuki, S. Kubo, K. Shizu, T. Fukushima, A. Wakamiya, Y. Murata, C. Adachi and H. Kaji, Angew. Chem., Int. Ed., 2015, 54, 15231.

38 I. S. Park, J. Lee and T. Yasuda, J. Mater. Chem. C, 2016, 4, 7911.

39 P. A. Vecchi, A. B. Padmaperuma, H. Qiao, L. S. Sapochak and P. E. Burrows, Org. Lett., 2006, 8, 4211.

40 C. Murawski, K. Leo and M. C. Gather, Adv. Mater., 2013, 25, 6801.

41 M. A. Baldo, C. Adachi and S. R. Forrest, Phys. Rev. B: Condens. Matter, 2000, 62, 10967.

42 C. Adachi, M. A. Baldo and S. R. Forrest, J. Appl. Phys., 2000, 87, 8049.

43 T. Takahashi, K. Shizu, T. Yasuda, K. Togashi and C. Adachi, Sci. Technol. Adv. Mater., 2014, 15, 034202. 